Pacific Journal of Mathematics

ON THE THEORY OF COMPACT OPERATORS IN YON 


\title{
ON THE THEORY OF COMPACT OPERATORS IN VON NEUMANN ALGEBRAS II
}

\author{
VICTOR KAFTAL
}

In their recent works $L$. Zsido' and P. A. Fillmore have extended Weyl's version of the classical Weyl-von Neumann theorem to infinite semi-finite countably decomposable von Neumann factors, by proving that for every self-adjoint operator $A$ in the factor there is a diagonal operator $B=$ $\sum \lambda_{n} E_{n}$ such that $A-B$ is compact, the $E_{n}$ are one-dimensional projections and $\left\{\lambda_{n}\right\}$ is dense in the essential spectrum of $A$. In this paper we extend the Weyl-von Neumann theorem in a different way.

First we extend the von Neumann version of the theorem to both finite and infinite factors by proving that $A-B$ can be chosen as a Hilbert-Schmidt operator of arbitrarily small norm. We have to drop the condition about the $\lambda_{n}$ or the dimension of the $E_{n}$.

In the second section we shall first re-obtain an equivalent form of Fillmore's theorem and then we shall generalize it to the case of normal operators, thus extending the Berg-Sikonia-Halmos theorem (see [2], [13], and [8]) to infinite factors. Finally we shall examine the possibility of choosing $B$ in the von Neumann algebra generated by $A$ and we shall generalize to normal operators a connected theorem by Zsido' [15].

We wish to thank M. Sonis for having called our attention to this problem.

1. The Weyl-von Neumann theorem in von Neumann factors. Let $H$ be a Hilbert space, $\mathscr{A}$ be a countably decomposable (i.e., $\sigma$ finite) semi-finite (i.e., type I or II) von Neumann factor on $H, \mathscr{A}^{\prime}$ be its commutant and $\mathscr{J}$ be the ideal of compact operators of $\mathscr{A}$, that is, the norm closure of the ideal of the operators $A \in \mathscr{A}$ with range projection $R_{A}$ finite relatively to $\mathscr{A}$ (finite operators for short).

Let $\operatorname{Tr}\left(\operatorname{Tr}^{\prime}\right)$ be a semi-finite faithful normal trace on $\mathscr{A}^{+}\left(\mathscr{A}^{\prime+}\right)$ and $D\left(D^{\prime}\right)$ be its restriction to the projections of $\mathscr{A}\left(\mathscr{A}^{\prime}\right)$. We use the normalization of the relative dimensions $D$ and $D^{\prime}$ for which $D(I)=1\left(D^{\prime}(I)=1\right)$ when $\mathscr{A}\left(\mathscr{A}^{\prime}\right)$ is finite and the linking constant $C_{\mathscr{A}}=1$ if $\mathscr{A}, \mathscr{A}^{\prime}$ or both are infinite.

Let $\mathscr{S}_{2}(\mathscr{A})$ be the Hilbert-Schmidt class of $\mathscr{A}$, i.e., the (generally incomplete) normed ideal of the operators $A \in \mathscr{A}$ for which 
$\|A\|_{2}=\left\{\operatorname{Tr}\left(A A^{*}\right)^{1 / 2}<\infty\right.$. Clearly $\mathscr{S}_{2}(\mathscr{A}) \subset \mathscr{F}$ and if $A$ is finite, then $\|A\|_{2} \leqq\left(D\left(R_{A}\right)\right)^{1 / 2}\|A\|$. Thus the classical inequality $\|A\| \leqq\|A\|_{2}$ does not hold for type II factors.

Let us finally note that while in the $L(H)$ case, or more general$1 y$, in the type I case, every finite self-adjoint operator is diagonal (i.e., has purely point spectrum), this is no longer true for type II factors. Hence in our generalization of the Weyl-von Neumann theorem, we shall consider finite factors too:

TheOREm 1.1. For $A=A^{*} \in \mathscr{A}$ and $\eta>0$ there is a diagonal operator $B=B^{*} \in \mathscr{A}$ such that $S=A-B \in \mathscr{S}_{2}(\mathscr{A})$ and $\|S\|<\eta$, $\|S\|_{2}<\eta$.

Maintaining a certain analogy with the von Neumann proof (see [11]), we first give the following lemma:

Lemma 1.2. Let $\mathscr{A}^{\prime}$ be finite and let $A=A^{*} \in \mathscr{A}$. Then for every $0 \neq f \in H$ and $\eta>0$ there is a finite projection $P$ and $a$ finite operator $S=S^{*}$ such that:

(1) $(I-P) f=0$

(2) $A-S$ is reduced by $P$

(3) $(A-S) P$ is diagonal

(4) $\|S\|<\eta$ and $\|S\|_{2}<\eta$.

Proof of the lemma. Let $n$ be an integer greater than $\max \left[3(3\|A\| / \eta)^{2}, 3\|A\| / \eta\right]$, let $\Delta_{j}=\left(\lambda_{j}-\|A\| / n, \lambda_{j}+\|A\| / n\right] j=1 \cdots n$ be a disjoint cover of $\sigma(A)$

and let $e_{j}=\left\{\begin{array}{ccc}\frac{E_{\Delta_{j}} f}{\left\|E_{\Delta_{j}} f\right\|} & \text { if } & E_{\Delta_{j}} f \neq 0 \\ 0 & \text { if } & E_{\Delta_{j}} f=0\end{array}\right.$

where $E$ is the spectral measure of $A$. Without loss of generality we may assume that $e_{j} \neq 0$ for $j=1 \cdots m \leqq n$. Let $E_{j}=E_{e j}\left(\mathscr{C}^{\prime}\right)$ be the smallest projection of $\mathscr{A}$ whose range contains $e_{j}$ and let $P=\sum_{j=1}^{m} E_{j}$. If $\mathscr{A}$ is finite then $D\left(E_{j}\right) \leqq 1$; if it is infinite then $C_{\mathscr{A}}=1$ and again $D\left(E_{j}\right)=D^{\prime}\left(E_{e_{j}}(\mathscr{A})\right) \leqq 1$. Thus $D(P) \leqq m \leqq n$. As $E_{j} \leqq E_{\Delta_{j}}$ we have $P f=\sum_{j=1}^{m} E_{j} E_{\Delta_{j}} f=\sum_{j=1}^{n} E_{\Delta_{j}} f=f$ (and hence (1)). The choice $S=A-\sum_{j=1}^{m} \lambda_{j} E_{j}-(I-P) A(I-P)$ clearly satisfies (2) and (3). As $S=P \sum_{j=1}^{m}\left(A-\lambda_{j} I\right) E_{j}+P A(I-P)+(P A(I-P))^{*}$ is the sum of three operators with rank not greater than the dimension of $P, D\left(R_{S}\right) \leqq 3 n$. Thus $S$ is self-adjoint, finite and hence Hilbert-Schmidt. As $(I-P) A P=(1-P) \sum_{j=1}^{m}\left(A-\lambda_{j} I\right) E_{j}$ we have $\|S\| \leqq 3\left\|\sum_{j=1}^{m}\left(A-\lambda_{j} I\right) E_{j}\right\|$. Moreover $E_{\Delta_{j}}$ reduces $\left(A-\lambda_{j} I\right) E_{j}$ and $\left\|\left(A-\lambda_{j} I\right) E_{\lrcorner_{j}}\right\| \leqq\|A\| / n$ hence for every $x \in H$, 


$$
\left\|\sum_{j=1}^{m}\left(A-\lambda_{j} I\right) E_{j} x\right\|^{2}=\sum_{j=1}^{m}\left\|\left(A-\lambda_{j} I\right) E_{\Delta_{j}} E_{j} x\right\|^{2} \leqq\left(\frac{\|A\|}{n}\|P x\|\right)^{2} .
$$

Therefore $\|S\| \leqq 3\|A\| / n<\eta$ and $\|S\|_{2} \leqq(3 n)^{1 / 2}(3\|A\| / n)<\eta \quad$ which completes the proof.

Proof of the theorem. It is known that every semi-finite (countably decomposable) factor is *-isomorphic to a (countably decomposable) factor with finite commutant (see Dixmier [3] Cor. 3 pg. 233). A *-isomorphism preserves the operator norm (see Dixmier [4] Prop. 1.3.7), the relative dimension up to a normalization constant (see Naimark [10] Th. 3. §6) and hence the Hilbert-Schmidt norm (up to a multiplicative constant), and the class of diagonal operators, (Dixmier [3] Cor. 1 pg. 54), hence we can prove the theorem with the additional condition that $\mathscr{A}^{\prime}$ is finite.

The countable decomposability of $\mathscr{A}$ provides us with a counta-

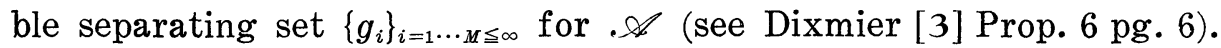
We are going to construct by induction on $n=1 \cdots N \leqq M$ a sequence $\left\{P_{n}\right\}$ of projections of $\mathscr{A}$ and a sequence $\left\{S_{n}\right\}$ of selfadjoint Hilbert-Schmidt operators such that
(1) $\left(I-\sum_{j=1}^{n} P_{j}\right) g_{i}=0$ for every $i \leqq n$
(2) $S_{n} P_{i}=0, P_{n} P_{i}=0$ for every $i<n$
(3) $\left(A-\sum_{j=1}^{n} S_{j}\right)$ is reduced by $P_{n}$
(4) $\left(A-\sum_{j=1}^{n} S_{j}\right) P_{n}$ is diagonal
(5) $\left\|S_{n}\right\|<2^{-n} \eta$ and $\left\|S_{n}\right\|_{2}<2^{-n} \eta$.

Let us apply the Lemma 1.2 to the vector $g_{1}$, the operator $A \in \mathscr{A}$ and the constant $2^{-1} \eta$. It is obvious that the resulting projection $P_{1}$ and self-adjoint Hilbert-Schmidt operator $S_{1}$ satisfy the conditions (1) to (5). Let us assume that we have found $\left\{P_{j}\right\},\left\{S_{j}\right\}$ for $j=$ $1 \cdots n$, satisfying all those conditions and that there is a first index $k_{n}, n+1 \leqq k_{n} \leqq M$ for which $\left(I-\sum_{j=1}^{n} P_{j}\right) g_{k_{n}} \neq 0$. Let $\Phi_{n}$ be the canonical *-isomorphism from $\mathscr{A}_{n}=\left(I-\sum_{j=1}^{n} P_{j}\right) \cdot \mathscr{A}\left(I-\sum_{j=1}^{n} P_{j}\right) \subset$ $\mathscr{A}$ onto the factor $\mathscr{A}_{n}=\mathscr{A}_{\left(I-\sum_{j=1}^{n} P_{j}\right)} . \Phi_{n}$ maps $\mathscr{S}_{2}(\mathscr{A}) \cap \mathscr{A}_{n}$ onto $\mathscr{S}_{2}\left(\mathscr{\mathscr { A }}_{n}\right)$ and there is a constant $d_{n}$ such that for every $B \in \mathscr{S}_{2}\left(. \Omega^{\prime}\right) \cap$ $\mathscr{A}_{n}$ we have $\left\|\Phi_{n}(B)\right\|_{2}=d_{n}\|B\|_{2}$.

As $\left(\tilde{\mathscr{A}}_{n}\right)^{\prime}$ is *-isomorphic to $\mathscr{A}^{\prime}$, it is finite, hence we can apply Lemma 2.1 to the vector $0 \neq\left(I-\sum_{j=1}^{n} P_{j}\right) g_{k_{n}}$, the self-adjoint operator $\Phi_{n}\left(\left(A-\sum_{j=1}^{n} S_{j}\right)\left(I-\sum_{j=1}^{n} P_{j}\right)\right) \in \tilde{\mathscr{A}}_{n}$ and the constant $\min \left(2^{-(n+1)} \eta\right.$, $\left.2^{-(n+1)} d_{n} \eta\right)$.

Let us call $P_{n+1}, S_{n+1} \in \mathscr{A}_{n}$ the images under $\Phi_{n}^{-1}$ of the resulting projection and Hilbert-Schmidt operator in $\tilde{\mathscr{A}_{n}}$. Clearly, $S_{n+1}$ is Hilbert- 
Schmidt in $\mathscr{A},\left\|S_{n+1}\right\|<2^{-(n+1)} \eta$ and $\left\|S_{n+1}\right\|_{2}=d_{n}^{-1}\left\|\Phi_{n}\left(S_{n+1}\right)\right\|_{2} \leqq 2^{-(n+1)} \eta$. Moreover $P_{n+1} P_{i}=0$ and $S_{n+1} P_{i}=0$ for every $i \leqq n$ and as $\left(I-P_{n+1}\right)\left(I-\sum_{j=1}^{n} P_{j}\right) g_{i}=\left(I-\sum_{j=1}^{n+1} P_{j}\right) g_{i}=0$ for every $i \leqq k_{n}$ we obtain (1), (2) and (5). As the image under $\Phi_{n}^{-1}$ of a diagonal operator in $\mathscr{A}_{n}$ is diagonal in $\mathscr{A}$ we easily obtain (3) and (4).

Thus we shall obtain by induction a sequence $\left\{P_{n}\right\}$ and $\left\{S_{n}\right\}$, $n=1 \cdots N \leqq M$ satisfying (1) to (5) such that $\left(I-\sum_{n=1}^{N} P_{n}\right) g_{i}=0$ for every $i$, i.e., such that $\sum_{n=1}^{N} P_{n}=I$. Let us call $B_{n}=$ $\left(A-\sum_{\jmath_{1}}^{n} S_{j}\right) P_{n}, \quad B=\sum_{n=1}^{N} B_{n} . \quad B=B^{*} \in \mathscr{A}$ and is diagonal as a direct sum of diagonal operators. Let us call $S=\sum_{n=1}^{N} S_{n}$. $S$ is Hilbert-Schmidt as the series converges (in the case of $N=\infty$ ) both in the operator norm and in the Hilbert-Schmidt norm. Clearly $\|S\|<\eta$ and $\|S\|_{2}<\eta$. As for every $i \leqq n,\left(A-\sum_{j=1}^{n} S_{j}\right) P_{i}=$ $\left(A-\sum_{j=1}^{i} S_{j}\right) P_{i}=B_{i}$ we have $A-\sum_{j=1}^{n} S_{j}=\sum_{i=1}^{n} B_{i}+\left(A-\sum_{j=1}^{n} S_{j}\right)$ $\left(I-\sum_{j=1}^{n} P_{j}\right)$ and hence $A-B=S$.

REMARK 1.3. It is impossible to extend this generalization of the Weyl-von Neumann theorem in the direction followed by Zsido' (Prop. 2.3 [15]) and by Fillmore (Th. 2.6 [7]), that is to request that the $\lambda_{n}$ belong to the essential spectrum of $A$. Indeed if $A \in \mathscr{J}$ then $\sigma^{e}(A)=\{0\}$ and hence $B$ has to be zero and $S=A$.

REMARK 1.4. It is possible to generalize Theorem 1.1 to the $\mathscr{S}_{p}(\mathscr{A})$ class with the \|\|$_{p}$ norm for every $p>1$ (see Ovchinnikov [12] $3^{\circ}$ : the $\mathscr{L}_{p}(\Gamma)$ space). It is enough to note that a ${ }^{*}$-isomorphism preserves the $\mathscr{S}_{p}$ classes and the $p$ norms (up to a multiplicative constant) and that for every finite operator $A$, $\|A\|_{p} \leqq D\left(R_{A}\right)^{1 / p}\|A\|$ : the proof of the lemma and of the theorem can then be carried over with only minor changes.

2. The Berg-Sikonian-Halmos theorem in von Neumann factors. Henceforth $\mathscr{A}$ will be a countably decomposable infinite semi-finite von Neumann factor (i.e., type $I_{\infty}$ or $I I_{\infty}$ ). Let $A$ be a diagonal operator $A=\sum_{n=1}^{N} \lambda_{n} E_{n}$ where we assume $\lambda_{i} \neq \lambda_{j}$ for $i \neq j$. Let us denote $\Lambda_{A}=\left\{\lambda_{n}\right\}_{n=1 \cdots N \leqq \infty}$ and $\mu_{A}\left(\lambda_{n}\right)=D\left(E_{n}\right)$. If $\mu_{A}\left(\lambda_{n}\right)=\infty$ for every $n=1 \cdots N$, then we say that $A$ is strongly diagonal.

Let us recall that as a diagonal operator $A$ is normal, all its five essential spectra coincide (see Prop. 3.7 Kaftal [9]) and that (see Prop. $3.8 \mathrm{ibid)} k$ belongs to the essential spectrum $\sigma^{e}(A)$ if and only if for every open set $S \ni k, E_{S}=\sum_{\lambda_{n} \in S} E_{n}$ is infinite $(E$ is the spectral measure of $A$ ). This leads us to the following characterization of the essential spectrum of a diagonal operator.

Proposition 2.1. Let $A \in \mathscr{A}$ be diagonal. Then $k \in \sigma^{e}(A)$ iff 
either $k \in \Lambda_{A}$ and $\mu_{A}(k)=\infty$ or there is a subsequence of $\Lambda_{A}, \lambda_{n_{j}} \rightarrow k$ such that $\sum_{j=h}^{\infty} \mu_{A}\left(\lambda_{n_{j}}\right)=\infty$ for every $h$.

Proof. If there is such a subsequence, then $\lambda_{n_{j}}$ is eventually in every open $S \ni k$ and hence $E_{S}$ is infinite.

On the other hand, if $k \in \sigma^{e}(A)$ and $k \notin \Lambda_{A}$ or $\mu_{A}(k)<\infty$ then for every open $S \ni k, \sum_{\lambda_{n} \in S \cap\left(A_{A}-\{k\}\right)} \mu_{A}\left(\lambda_{n}\right)=\infty$. Thus we can construct a nested sequence of open circles $S_{m}$ with center $k$ and radius $r_{m} \rightarrow 0$ such that $S_{m}-S_{m+1} \ni\left\{\lambda_{n_{j}}\right\}_{j=j_{m}+1}, \cdots, j_{m+1} \subset\left(\Lambda_{A}-\{k\}\right)$ and $\sum_{\jmath_{=j_{m}+1}}^{j_{m+1}} \mu_{A}\left(\lambda_{n_{j}}\right) \geqq$ 1. Then $\lambda_{n_{j}} \rightarrow k$ and $\sum_{j=h}^{\infty} \mu_{A}\left(\lambda_{n_{j}}\right)=\infty$ for every $h$.

Let us note that if an operator is strongly diagonal then its spectrum coincides with its essential spectrum. The converse is of course false-consider any nonnormal nilpotent operator.

If a normal operator $A$ has a one point essential spectrum $\sigma^{e}(A)=\{k\}$ then $\sigma^{e}(A-k I)=\{0\}$ and by Proposition 3.9. Kaftal [9], $A-k I \in \mathscr{F}$ i.e., $A$ is strongly diagonal $\bmod \mathscr{F}$.

This remains true even if $\sigma^{e}(A)$ is infinite: we shall prove it for self-adjoint operators, and then extend it to normal operators. First we need the following technical lemma:

Lemma 2.2. Let $A=A^{*} \in \mathscr{A}, E$ be the spectral measure of $A$ and $M \subset R$ be a Borel set. Then $\sigma^{e}\left(A E_{M}\right) \subset\left(\sigma^{e}(A) \cap \bar{M}\right) \cup\{0\} . \quad I f$ $\sigma^{e}(A) \cap \bar{M}=\{k\}$ then $A E_{M}-k E_{M} \in \mathscr{F}$. If moreover $M$ is an interval and its measure is $\mu(M)$ then $\left\|A E_{M}-k E_{M}\right\| \leqq \mu(M)$.

Proof. First let us recall that $\sigma^{e}\left(A E_{M}\right) \subset \sigma\left(A E_{M}\right) \subset \bar{M} \cup\{0\}$ (see Cor. 6.X.2. [5] Dunford and Schwartz). Let $0 \neq \lambda \in \sigma^{e}\left(A E_{M}\right)$ and let $\Delta$ be any open interval containing $\lambda$. We can assume that $\Delta \nexists 0$. If we call $F$ the spectral measure of $A E_{M}$ we have $F_{\Delta}=E_{\Delta \cap M} \leqq E_{\Delta}$ hence $E_{\lrcorner}$is infinite. Thus $\lambda \in \sigma^{e}(A)$. Let $M-k\left(\sigma^{e}(A)-k\right)$ be the translation of $M\left(\sigma^{e}(A)\right)$ by $-k$. It is easy to see that $\sigma^{e}\left((A-k I) E_{M}\right) \subset$ $\left(\sigma^{e}(A)-k \cap \overline{M-k}\right) \cup\{0\}$. Thus if $\sigma^{e}(A) \cap \bar{M}=\{k\}$ then $\sigma^{e}\left((A-k I) E_{M}\right) \subset$ $\{0\}$ and as it is nonvoid and we deal with self-adjoint operators, we have $A E_{M}-k E_{M} \in \mathscr{F}$ (see Th. 3.2. and Prop. 3.9. [9]). The last statement then follows by standard computations.

Proposition 2.3. Every self-adjoint operator of . 2 is strongly diagonal $\bmod \mathscr{F}$.

Proof. Because of Theorem 1.1 we can assume that $A$ is diagonal, i.e., $A=\sum_{n=1}^{N} \lambda_{n} E_{n}$. The case $N<\infty$ is trivial, so we can assume $N=\infty$. Let $k_{1}$ be the element of $\sigma^{e}(A)$ closest to $\lambda_{1}$. Let $\Delta_{1} \subset[-\|A\|,\|A\|]$ be the greatest interval containing $k_{1}$ such that 
for every $x \in \Delta_{1},\left|x-k_{1}\right|=\min _{k \in \sigma^{e}(A)}|x-k|$. If $E_{\Delta_{1}}=\sum_{n_{n} \in \Delta_{1} \cap A_{A}} E_{n}$ is infinite then let $\widetilde{\Lambda}_{1}=\varnothing$. If $E_{A_{1}}$ is finite, then, because of Proposition 2.1 we can construct a subsequence $\widetilde{\Lambda}_{1}=\left\{\lambda_{n}^{(1)}\right\} \subset \Lambda_{A}-\Delta_{1}$ such that $\lambda_{n}^{(1)} \rightarrow k_{1}$, $\sup _{n=1 \ldots \infty}\left|\lambda_{n}^{(1)}-k_{1}\right|<2^{-1}$ and $E_{\widetilde{A}_{1}}=\sum_{\lambda_{n} \in \tilde{A}_{1}} E_{n}$ is infinite.

Let $\Lambda_{1}=\left(\Delta_{1} \cap \Lambda_{1}\right) \cup \widetilde{\Lambda}_{1}, A_{\Lambda_{1}}=\sum_{\lambda_{n} \in \Lambda_{1}} \lambda_{n} E_{n}, E_{\Lambda_{1}}=\sum_{\lambda_{n} \in \Lambda_{1}} E_{n}$ and $K_{1}=$ $A_{\Lambda_{1}}-k_{1} E_{A_{1}}$. Clearly $E_{\Lambda_{1}}$ is infinite, $K_{1}=A E_{\Delta_{1}}-k_{1} E_{\Delta_{1}}+\sum \lambda_{n} \in \tilde{A}_{1}\left(\lambda_{n}-k_{1}\right) E_{n}$ belongs to $\mathscr{F}$ by Lemma 2.2, Proposition 2.1 here and Proposition 3.9 in [9] and $\left\|K_{1}\right\| \leqq \mu\left(\Delta_{1}\right)+2^{-1}$ by Lemma 2.2 .

Now we can consider the subsequence $\Lambda_{A}-\Lambda_{1}$ of $\Lambda_{A}$ and repeat the same construction. Then either the process stops after a finite number of steps (iff $\sigma^{e}(A)$ is finite) and the theorem is easily proved, or it is possible to construct by induction a sequence of disjoint intervals $\Delta_{j} \subset[-\|A\|,\|A\|]$ and of disjoint subsequences $A_{j} \subset A_{A}$ with $\Lambda_{A}=\bigcup_{j=1}^{\infty} A_{j}$ such that $A_{A_{j}}=k_{j} E_{A_{j}}+K_{j}, E_{A_{j}}$ is infinite, $K_{j} \in \mathscr{F}$ and $\left\|K_{j}\right\| \leqq 2^{-j}+\mu\left(\Delta_{j}\right)$. Clearly $A=\sum_{j=1}^{\infty} A_{\Lambda_{j}}=\sum_{j=1}^{\infty} k_{j} E_{\Lambda_{j}}+K$ where $\mathscr{I} \ni K=\sum_{j=1}^{\infty} K_{j}$ because as $\sum_{j=1}^{\infty}\left\|K_{j}\right\| \leqq 1+\mu[-\|A\|,\|A\|]$ the series converges in the norm topology.

It is easy to see by using Theorem 2.7 that Proposition 2.3 is essentially equivalent to Fillmore's Theorem 2.6 [7] (which however has been proved for separable Hilbert spaces).

In order to extend Proposition 2.3 to the normal operators of . $f$ we shall strengthen a result by Halmos [8].

Lemma 2.4. Let $C$ be a normal operator and let $W(C)$ be the von Neumann algebra generated by $C$. Then $C$ is a continuous function of a self-adjoint operator $A \in W(C)$.

Proof. Let $U$ be an isometric *-isomorphism from $W(C)$ onto $L^{\infty}(Z, \nu)$ where $Z$ is a locally compact space, $\nu$ is a positive measure with support $Z$ and $L^{\infty}(Z, \nu)$ is the $C^{*}$ algebra of $\nu$ measurable, essentially bounded complex valued functions on $Z$ (see Dixmier [3] Th. 1 pg. 118). Let $\phi$ be a continuous mapping from the Cantor set $\Gamma \subset[0,1]$ onto the compact set $\sigma(C)$ (which contains the closure of the essential range of $U C$, see Corollary X.2.9 by Dunford and Schwartz [5]). Halmos [8] has proved that $\phi$ has a Borel cross section $\psi: \sigma(C) \rightarrow \Gamma(\phi \circ \psi=1)$, hence $\psi \circ U C \in L^{\infty}(Z, \nu)$. Let $A=$ $U^{-1}(\psi \circ U C) \in W(C) . \quad A$ is self-adjoint since $\psi \circ U C$ is real valued, and clearly $\dot{\phi}(A)$ is well defined. Let $P_{n}$ be a sequence of complex valued polynomials converging uniformly to $\phi$. Then $P_{n}(A) \rightarrow \phi(A)$ in the norm topology of $W(A)$ and thus $U P_{n}(A) \rightarrow U \dot{\phi}(A)$ in the norm topology of $L^{\infty}(Z, \nu)$. But $U P_{n}(A)=P_{n} \circ U A=P_{n} \circ \psi \circ U C$ converges in that topology to $\phi \circ \psi \circ U C=U C$ hence $C=\phi(A)$. 
THEOREM 2.5. Every normal operator of . . is strongly diagonal $\bmod \mathscr{F}$.

Proof. Let $C$ be a normal operator of $\mathscr{A}$. According to the previous lemma $C=\phi(A)$ for some $\phi$ continuous on $\sigma(A)$ and $A=$ $A^{*} \in W(C) \subset \mathscr{A}$. We can apply Proposition 2.3 to $A$ and find a strongly diagonal self-adjoint $B=\sum_{n=1}^{N} \lambda_{n} E_{n} \in \mathscr{A}$ such that $A-B \in \mathscr{F}$. As $\sigma(B)=\sigma^{e}(B)=\sigma^{e}(A) \subset \sigma(A), \phi(B)=D \in \mathscr{L}$ is well defined and we have $D=\sum_{n=1}^{N} \phi\left(\lambda_{n}\right) E_{n}$, hence $D$ is strongly diagonal. Let $P_{n}$ be a sequence of complex valued polynomials converging uniformly to $\phi$. Clearly $P_{n}(A) \rightarrow \phi(A)=C, \quad P_{n}(B) \rightarrow \phi(B)=D$ in the norm topology and as $P_{n}(A)-P_{n}(B) \in \mathscr{F}$ we have $C-D \in \mathscr{F}$.

This theorem is the extension to von Neumann factors of the Halmos version of the Berg-Sikonia-Halmos theorem. (See [2], [13], [8].) The first two authors' version is slightly stronger as it asks that $\|C-D\|<\eta$ for any arbitrary $\eta>0$. As we have noted already in Remark 1.3 we cannot however strengthen our results in this direction. We can however proceed in the direction of von Neumann's inverse of Weyl's theorem, or more precisely, since we are dealing with normal operators, we can extend to von Neumann factors the Sikonia-Edgar, Ernest and Lee theorem. (See Th. 1 [13], Cor. 5.5 [6].) Let us first note that unitarily equivalent operators have the same essential spectrum. Indeed, the extension of the Weyl theorem to a semi-finite von Neumann algebra $\mathscr{B}$ (see Th. 3.3 [9]), states that if $A, B \in \mathscr{B}, A-B \in \mathscr{J}$ then $\sigma^{e}(A)=\sigma^{e}(B)$ for any of the essential spectra defined in $\S 3$ ibid. Moreover, if $U \in \mathscr{B}$ is unitary then $\sigma^{e}(A)=\sigma^{e}\left(U A U^{*}\right)$ because $U$ is Fredholm index zero and (by Th. 2.13 ibid.) $U(A-\lambda I) U^{*}=U A U^{*}-\lambda I$ is (left), (right), Fredholm or has index zero together with $A-\lambda I$. Thus we have

Proposition 2.6. Let .S be an infinite semi-finite von Neumann algebra and $\sigma^{e}$ be any of the essential spectra. If $A, B \in \mathscr{B}$ are unitarily equivalent mod. $\mathscr{T}$ then $\sigma^{e}(A)=\sigma^{e}(B)$.

The converse of this theorem is generally false for nonnormal operators. (Sikonia [13] and Edgar, Edgar, and Lee Th. 5.7 [6] for the case $=L(H)$.)

THEOREM 2.7. Let. $\mathscr{\text { L }}$ be a countably decomposable infinite semifinite von Neumann factor and let $A, B \in \mathscr{A}$ be normal and such that $\sigma^{e}(A)=\sigma^{e}(B)$. Then $A$ and $B$ are unitarily equivalent mod $\mathscr{F}$.

It is possible to carry over to this case the classical von Neumann 
proof (see Satz II [11]) which uses the diagonalizability of $A, B \bmod \mathscr{F}$ and a theorem on sequences having the same set of cluster points. The only difference with the classical case is that here it is necessary to take care that the diagonal operators $A^{\prime}=\sum_{n=1}^{N} \lambda_{n} E_{n}$, $B^{\prime}=\sum_{m=1}^{M} \gamma_{m} F_{m}$ such that $A-A^{\prime} \in \mathscr{F}, B-B^{\prime} \in \mathscr{J}$ have a decomposition for which $\mu_{A^{\prime}}\left(\lambda_{n}\right) \equiv \mu_{B^{\prime}}\left(\gamma_{m}\right)$ but this is always possible because of Theorem 2.5. This theorem has been obtained by Zsido' (see Cor. 3.3 [15]) as a corollary of a unitary implementation theorem for isomorphisms.

We can further use Lemma 2.4 to extend to normal operators a proposition proved for self-adjoint operators by Zsido' (see Prop. 2.1 [15]). Here $M$ is a von Neumann algebra, $\gamma$ a norm-closed two sided ideal in $M$, and $\Pi$ is the canonical homomorphism of $M$ onto $M / \gamma$.

Proposition 2.8. For every normal $C \in M$ there is a normal $D \in W(C)$ such that $C-D \in \gamma$ and $\sigma(D)=\sigma(\Pi D)$.

Proof. Let $C=\dot{\phi}(A)$ with $\dot{\phi}$ continuous on $\sigma(A)$ and $A=A^{*} \epsilon$ $W(C) \subset M$. We can apply to $A$ the above mentioned proposition by Zsido' and find in $W(A) \subset W(C)$ a self-adjoint $B$ such that $A-B \in \gamma$ and $\sigma(B)=\sigma(\Pi B)$. As $\sigma(B) \subset \sigma(A)$ we can define $D=\phi(B)$. Clearly $D \in W(A)$, hence $D \in W(C)$ and $\sigma(D)=\phi(\sigma(B))=\phi(\sigma(\Pi B))=\sigma(\phi(\Pi B))=$ $\sigma(\Pi D)$. The first and the third equalities follow from the usual spectral mapping theorem (see Dixmier [4] pg. 11) and the fourth one from Proposition 1.5.3 ibid.

In view of this proposition applied to $M=\mathscr{A}, \gamma=\mathscr{J}$ and of the fact that for $C$ and $D$ as in Theorem $2.5 \sigma(D)=\sigma^{e}(D)$, it is natural to inquire if the strongly diagonal $D$ can be chosen in $W(C)$. If $C=\phi(A)$ is diagonal then $A=A^{*}$ is diagonal too and by considering the construction given in Proposition 2.3 of the strongly diagonal $B=B^{*}$ such that $A-B \in \mathscr{J}$ we see that $B \in W(A) \subset W(C)$ and hence $D=\phi(B) \in W(C)$. If $\sigma^{e}(C)=\left\{\lambda_{j}\right\}_{j=1 \cdots_{n<\infty}}$ (iff $C$ is polynomially compact: same proof as Theorem 6.4 by Berberian [14]), $E$ is the spectral measure of $C$ and $S_{j} \ni \lambda_{j}$ for $j=1 \cdots n$ is an open disjoint cover of $\sigma^{e}(C)$, then $D=\sum_{j=1}^{n} \lambda_{j} E_{S_{j}} \in W(C)$ and $C-D \in \mathscr{F}$.

However in the general case this fails to hold as shown by the following example.

EXAMPLE 2.9. Let $\mu$ be a finite positive regular measure defined on the Borel sets of $X=[0,1]$, let $\mathscr{A}$ be the algebra of all bounded operators on $L^{2}(X, \mu)$ and let $A \in \mathscr{A}$ be the "multiplication by $x$ " and $E$ be the spectral measure of $A$. Every projection $P \in W(A)$ is a spectral projection of $A$, i.e., $P=E_{M}$ for some Borel set $M$, hence 
if $P \neq 0$ then $P$ is infinite. If there were a self-adjoint diagonal $D \in W(A)$ such that $A-D \in \mathscr{F}$ then there would be a $\lambda \neq 0$ and a Borel set $M$ such that $E_{M} \neq 0$ and $A E_{M}-\lambda E_{M} \in \mathscr{F}_{\text {. But this would }}$ lead to a contradiction. Indeed let $A_{n} \ni \lambda$ be a nested sequence of open intervals converging to $\{\lambda\}$. For every $n$ we have $A E_{M-\Delta_{n}}-$ $\lambda E_{M-\Delta_{n}} \in \mathscr{J}$ and hence $E_{M-\Delta_{n}}=0$ because otherwise $E_{M-\Delta_{n}}$ would be infinite and $0 \neq \lambda \in \sigma^{e}\left(A E_{M-\Delta_{n}}\right) \subset\left(\sigma^{e}(A) \cap \overline{M-\Delta_{n}}\right) \cup\{0\}$ (by Lemma 2.2) would imply $\lambda \notin \Delta_{n}$. Thus $E_{M}=E_{M \cap A_{n}}=E_{M} E_{\Delta_{n}} \rightarrow E_{M M} E_{[\lambda]}=0$ and we have $E_{M}=0$ against the assumption.

\section{REFERENCES}

1. S. K. Berberian, The Weyl spectrum of an operator, Indiana Univ. Math. J., 20 (1970), 529-554.

2. I. D. Berg, An extension of the Weyl-von Neumann theorem to normal operators, Trans. Amer. Math. Soc., 160 (1971), 365-371.

3. J. Dixmier, Les Algebres d'Operateurs dans l'Espace Hilbertien, 2nd ed. Paris: Gauthier-Villars, 1969.

4. — Les $C^{*}$-Algebres et Leurs Representations, 2nd ed. Paris: Gauthier-Villars, 1969.

5. N. Dunford and J. T. Schwartz, Linear Operators, Part II, New York-London-Sydney Interscience, 1967.

6. G. Edgar, J. Ernest, and S. G. Lee, Weighing operator spectra, Indiana University, Math. J., 21 (1971), 61-80.

7. P. A. Fillmore, Extensions relative to semi-finite factors, Rome conference, September, 1975.

8. P. R. Halmos, Continuous functions of Hermitian operators, Proc. Amer. Math. Soc., 31 (1972), No. 1, 130-132.

9. V. Kaftal, On the theory of compact operators in von Neumann algebras I, Indiana Univ. Math. J., 26 (1977), 447-457.

10. M. A. Naimark, Normed Rings, 1st Amer. ed. Wolters-Noordhoff, Groningen, 1970.

11. J. Von Neumann, Charakterisierung des Spektrums eines Integraloperators, Paris: Herman \& C., 1935.

12. V. I. Ovchinnikov, Symmetric spaces of measurable operators, Soviet Math. Dokl., 11 (1970), No. 2, 448-451.

13. W. Sikonia, The von Neumann converse of Weyl's theorem, Indiana Univ. Math. J., 21 (1971), 121-124.

14. H. Weyl, Ueber beschrankte quadratische Formen, deren Differenz vollstetig ist., Rend. Circ. Mat. Palermo, 27 (1909), 373-392.

15. L. Zsido', The Weyl-von Neumann theorem in semi-finite factors, J. Functional Anal., 18 (1975), 60-72.

Received July 11, 1977. This article is part of the author's thesis work at the TelAviv University.

Tel Aviv UnIversity

Tel Aviv, Israel 



\section{PACIFIC JOURNAL OF MATHEMATICS}

EDITORS

RICHARD ARENS (Managing Editor)

University of California

Los Angeles, California 90024

C. W. Curtis

University of Oregon

Eugene, OR 97403

C. C. MOORE

University of California

Berkeley, CA 94720
J. DUGUNDJI

Department of Mathematics University of Southern Californı Los Angeles, California 90007

R. Finn and J. Milgram Stanford University Stanford, California 94305

\section{ASSOCIATE EDITORS}

E. F. BECKENBACH

B. H. NEUMANN

F. WOLF

K. YoSHIDA

\section{SUPPORTING INSTITUTIONS}

UNIVERSITY OF BRITISH COLUMBIA
CALIFORNIA INSTITUTE OF TECHNOLOGY
UNIVERSITY OF CALIFORNIA
MONTANA STATE UNIVERSITY
UNIVERSITY OF NEVADA, RENO
NEW MEXICO STATE UNIVERSITY
OREGON STATE UNIVERSITY
UNIVERSITY OF OREGON

UNIVERSITY OF SOUTHERNEALIFORNIA STANFORD UNIVERSITY UNIVERSITY OF HAWAII UNIVERSITY OF TOKYO UNIVERSITY OF UTAH WASHINGTON STATE UNIVERSITY UNIVERSITY OF WASHINGTON 


\section{Pacific Journal of Mathematics \\ Vol. 79 , No. 1 \\ May, 1978}

Teófilo Abuabara, A remark on infinitely nuclearly differentiable

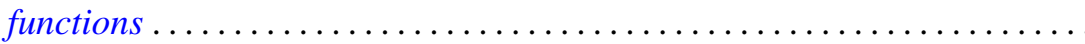

David Fenimore Anderson, Projective modules over subrings of $k[X, Y]$

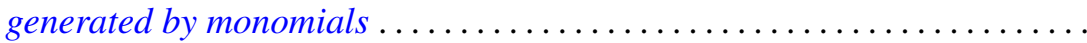

Joseph Barback and Thomas Graham McLaughlin, On the intersection of

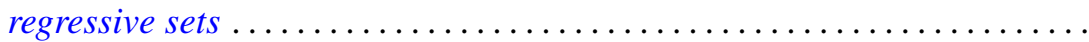

Murray Bell, John Norman Ginsburg and R. Grant Woods, Cardinal inequalities for topological spaces involving the weak Lindelof

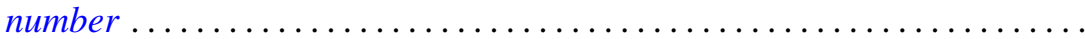

Laurence Richard Boxer, The space of ANRs of a closed surface ............

Zvonko Cerin, Homotopy properties of locally compact spaces at

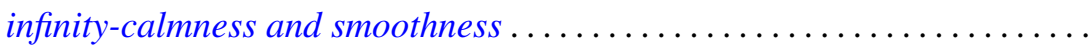

Isidor Fleischer and Ivo G. Rosenberg, The Galois connection between partial functions and relations..................................

John R. Giles, David Allan Gregory and Brailey Sims, Geometrical implications of upper semi-continuity of the duality mapping on a Banach

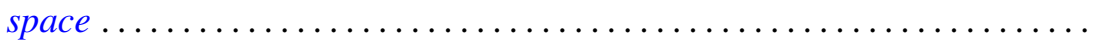

Troy Lee Hicks, Fixed-point theorems in locally convex spaces ............ Hugo Junghenn, Almost periodic functions on semidirect products of transformation semigroups ........................

Victor Kaftal, On the theory of compact operators in von Neumann algebras. II . . . .

Haynes Miller, A spectral sequence for the homology of an infinite

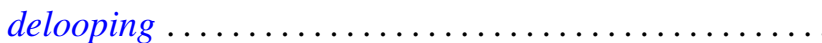

Sanford S. Miller, Petru T. Mocanu and Maxwell O. Reade, Starlike integral operators...

Stanley Stephen Page, Regular FPF rings ...............

Ghan Shyam Pandey, Multipliers for C, 1 summability of Fourier series ...

Shigeo Segawa, Bounded analytic functions on unbounded covering surfaces...

Steven Eugene Shreve, Probability measures and the C-sets of

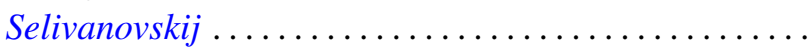

Tor Skjelbred, Combinatorial geometry and actions of compact Lie groups....

Alan Sloan, A note on exponentials of distributions.

Colin Eric Sutherland, Type analysis of the regular representation of a nonunimodular group.

Mark Phillip Thomas, Algebra homomorphisms and the functional

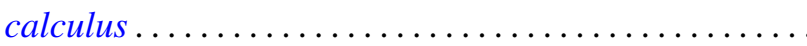

Sergio Eduardo Zarantonello, A representation of $H^{p}$-functions with

$0<p<\infty$. 